\title{
Full-length osteopontin and its splice variants as modulators of chemoresistance and radioresistance (Review)
}

\author{
ETEL R.P. GIMBA ${ }^{1,2}$, MARIANA C.M. BRUM ${ }^{1}$ and GABRIELA NESTAL DE MORAES ${ }^{3}$ \\ ${ }^{1}$ Program of Cellular and Molecular Oncobiology, National Cancer Institute, Rio de Janeiro 20231-050; \\ ${ }^{2}$ Department of Natural Sciences, Fluminense Federal University, Rio das Ostras 28880-000; \\ ${ }^{3}$ Cellular and Molecular Hemato-Oncology Laboratory, Molecular Hemato-Oncology Program, \\ National Cancer Institute, Rio de Janeiro 20230-130, Brazil
}

Received August 31, 2018; Accepted October 25, 2018

DOI: $10.3892 /$ ijo.2018.4656

\begin{abstract}
Osteopontin (OPN) is a matricellular phosphoglycoprotein overexpressed in several tumor types and can activate several aspects of cancer progression in solid and non-solid tumors. In the present review, the roles of OPN in mediating resistance to chemotherapy and radiotherapy and their main associated signaling pathways were summarized and discussed. Furthermore, it was detailed how OPN expression may be able to modulate resistance to these therapies by controlling epithelial cell plasticity, stemness potential and cell survival. Based on these data, the use of OPN and associated signaling was then proposed as potential molecular targets in order to sensitize resistant cells to main current therapeutic approaches. Finally, based on experimental evidence obtained by our group, the importance of investigating the specific roles OPN splicing isoforms have and how their properties may specifically control resistance to therapy was highlighted. These data elucidate a better understanding of how total OPN and their splicing isoforms, as well as their associated signaling, may contribute to main aspects of chemoresistance and radioresistance, such as those controlling cell survival, apoptosis, autophagy, stemness, epithelial cell plasticity and associated cell receptors.
\end{abstract}

\section{Contents}

1. Introduction

2. OPN and resistance to chemotherapy

3. OPN and association to radioresistance

4. OPN in the interface of epithelial plasticity and therapeutic resistance

Correspondence to: Dr Etel R.P. Gimba, Department of Natural Sciences, Fluminense Federal University, 1-7 Recife Street, Bela Vista, Rio das Ostras 28880-000, Brazil

E-mail: etelgimba@id.uff.br

Key words: osteopontin, chemoresistance, radioresistance, splicing isoforms, signaling pathways
5. OPN as a target to overcome resistance to cancer therapy

6. OPN splice variants and their potential role in tumor resistance

7. Conclusions

\section{Introduction}

Cancer therapy resistance is mediated by several mechanisms, including intrinsic and extrinsic factors, and those originating from the tumor microenvironment (TME) (1). Most of the widely used chemotherapeutic agents and $\gamma$-radiation utilize apoptosis or autophagy as common death pathways. Thus, a better understanding of the molecular mechanisms behind tumor biology and cancer therapy resistance is a mandatory step to propose novel approaches aiming to bypass chemotherapy and radiotherapy resistance and associated gene products. Among several markers associated with response to therapy, osteopontin (OPN) has been identified as a key molecule $(2,3)$.

OPN is a multi-functional chemokine-like matricellular phosphoglycoprotein. Depending on its intracellular or extracellular localization, OPN is involved in a series of physiological roles, including inflammation, cell adhesion and migration, differentiation, cell survival and apoptosis, as well as regulation of bone matrix mineralization. These diverse biological roles are partly due to its capacity to interact with several molecules, including cell surface receptors, such as integrin and cluster of differentiation (CD44), intracellular signaling molecules, calcium and heparin. OPN is produced by distinct cell types, such as epithelial, stromal, immune system, bone and endothelial cells (4). High OPN expression has also been detected in adipose tissues and body fluids (4).

In multiple cancer types, OPN expression is upregulated (3). In tumors, OPN regulates tumorigenesis, tumor progression and metastasis formation (5), by activating cell migration, inhibiting apoptosis (6,7), stimulating angiogenesis (8) and metabolism (9), and modulating the tumor microenvironment (10) and the immune system (11). Notably, OPN can also promote cell survival by negatively regulating apoptosis in response to stress conditions, including exposure to anticancer agents effect (12). OPN performs these roles by binding to cell surface receptors, including integrin and CD44 cell receptors (13). 
Several aberrantly activated signaling pathways can activate OPN expression in cancer cells, such as activator protein-1, mycA, phosphatidylinositol 3 kinase (PI3K), serine/threonine kinase (AKT) and nuclear factor-қB (NF-қB) $(14,15)$. Notably, OPN has been described as a key modulator of cancer hallmarks, by regulating the main aspects of tumor progression in several tumor models $(16,17)$. OPN is able to promote tumor cell migration by interacting with integrin receptors, especially $\alpha v \beta 3$ integrin, stimulating cell adhesion and tumor cell migration properties (3). It has been demonstrated previously that OPN downregulation in breast cancer cell lines inhibits OPN interaction with $\alpha v \beta 3$ integrin, thereby impairing cell migration, invasion and apoptosis (18). It was demonstrated that these effects have been mediated by PI3K/AKT/mechanistic target of rapamycin (mTOR) signaling, promoting the upregulation of light chain 3 and beclin-3, then favoring apoptotic cell death, while inhibiting aggressive phenotype of these breast cancer cells. It has also been demonstrated that alteration of OPN expression levels may influence tumor growth, migration and cell cycle in human nasopharyngeal CNE-2 carcinoma cell lines (18). When downregulating OPN expression, diminished levels of matrix metalloproteinase MMP-2 and MMP-9 have been observed, evidencing that OPN may also induce MMP expression levels through the activation of NF-қB signaling in this tumor model (19). OPN expression levels can also modulate mitochondrial mediated apoptotic cell death, involving cytochrome $c$, apoptotic protease activating factor 1 , cleaved caspase-3 and B cell lymphoma $(\mathrm{Bcl})-2 / \mathrm{Bcl}-2$-associated $\mathrm{X}$ protein, resulting in lower expression levels of proteins associated with cell invasion, such as MMP-2 and urokinase-type plasminogen activator (uPA) (20). Then, downregulation of OPN expression levels can promote apoptotic cell death and cell invasion properties in a mitochondrial-dependent pathway (20). OPN can also promote tumorigenesis and tumor progression by evading apoptotic cell death, mainly by interacting with CD44 cell receptors (21). Furthermore, OPN interactions with immune and inflammatory cells from the TME perform essential roles on tumor development and progression. Besides being produced by tumor cells, OPN can also be secreted by stromal and infiltrating inflammatory cells that can affect the TME and its corresponding cell roles. Stromal fibroblasts can also be influenced by OPN $(22,23)$. Given its roles in angiogenesis, extracellular matrix remodeling and metastasis, their presence and influence by OPN can also favor tumor growth $(22,23)$. It has been reported that when interacting with $\alpha 9 \beta 1$, OPN activates p38 and extracellular signal-regulated kinase (ERK) signaling, which then can promote the expression of cyclooxygenase-2 and prostaglandin E (PGE), favoring melanoma tumor cell migration (24). In addition, macrophages from the TME, when activated by OPN can promote angiogenesis via PGE2 and stimulate the expression of MMP-9, consequently promoting tumor progression in this tumor model (24). When recruiting macrophages to the tumor inflammatory environment, OPN can also stimulate tumorigenesis (25).

OPN also induces the expression and activity of MMPs, which can contribute to tumor metastasis by degrading the extracellular matrix (ECM) and promoting cell invasion. Conversely, OPN biological activity can also be modulated by MMP-induced cleavage (26). It is well-known that OPN stimulates tumor cell invasion and migration possibly by inhibiting apoptotic cell death and by regulating the activity of MMP-2 and MMP-9 that degrade the ECM (27). OPN can stimulate the activity of MMP-9, modulating multiple signaling pathways, such as focal adhesion kinase (FAK), ERK and NF-қB, which then can regulate cytoskeleton architecture, cell growth, motility and extracellular matrix $(\mathrm{ECM})(28,29)$. By activating PI3K/AKT signaling, OPN can similarly regulate hypoxia-inducible factor (HIF)- $1 \alpha$ expression via $\alpha v \beta 3$ integrin interaction and promoting ECM degradation through UPA and MMP-9, further mediating metastasis formation in ovarian cancer cells (30).

Also in the context of OPN roles on modulating the TME and the immune system, OPN has been described as a multifactorial cytokine activated by $\mathrm{T}$ lymphocytes, monocytes, macrophages, epithelial cells, fibroblasts and a promoter of cell-mediated immune responses $(15,28)$.

Similarly, OPN is a typical angiogenesis stimulating factor, sustaining tumor progression and metastatic growth. The role of OPN in angiogenesis is mainly associated with OPN interaction with $\alpha v \beta 3$ integrin, a central angiogenesis marker (31), but is also associated with several other factors, including vascular endothelial growth factor (VEGF) $(32,33)$. OPN interactions with VEGF are also mediated by aberrant signaling pathways, such as PI3K/AKT and ERK1/ERK2 $(32,33)$. It has also been demonstrated in acute leukemia that the expression of OPN and VEGF are strongly correlated with the occurrence and development of this non-solid tumor. In this model, OPN can regulate VEGF expression and promote angiogenesis besides favoring disease progression (31). It was also found that OPN interaction with $\alpha v \beta 3$ activates signaling pathways, such as breast tumor kinase/NF-қB/activating transcription factor (TF)-4, promoting cell migration, tumor growth, endothelial cell migration and angiogenesis (34). Our group also demonstrated that $\mathrm{OPNb}$ and OPNc splicing isoforms favored tumor growth, cell proliferation, invasion and migration by modulating VEGF, MMP-2 and MMP-9 expression levels through PI3K signaling (35).

It has also been demonstrated that OPN can modulate metabolism by signaling through the activation of oxidoreductase gene expression, associated with the mitochondrial respiratory chain, the hexose monophosphate shunt or the regulation of the hexose monophosphate shunt (9). Furthermore, it has been reported that OPN can disrupt liver cholesterol metabolism (36).

The majority of the data regarding the role of OPN in cancer cell refers to total OPN, which includes the sum of all OPN isoforms, including those generated by alternative splice, post-translational modifications and alternative translation $(17,37)$.

OPN primary transcript suffers alternative splicing, generating at least five splice variants, named OPNa (which contain all coding exons), OPNb (exon 5 is deleted), OPNc (exon 4 is deleted), OPN4 (in which both exon 4 and exon 5 are deleted) and OPN5 (which contains an additional exon originated from inclusion of a region from intron 3; Fig. 1). OPN splicing isoforms (OPN-SI) are aberrantly expressed in cancer cells (17). Particularly, the expression and functional roles of OPNa, OPNb and OPNc have been broadly studied in distinct tumor models, in which they were demonstrated 


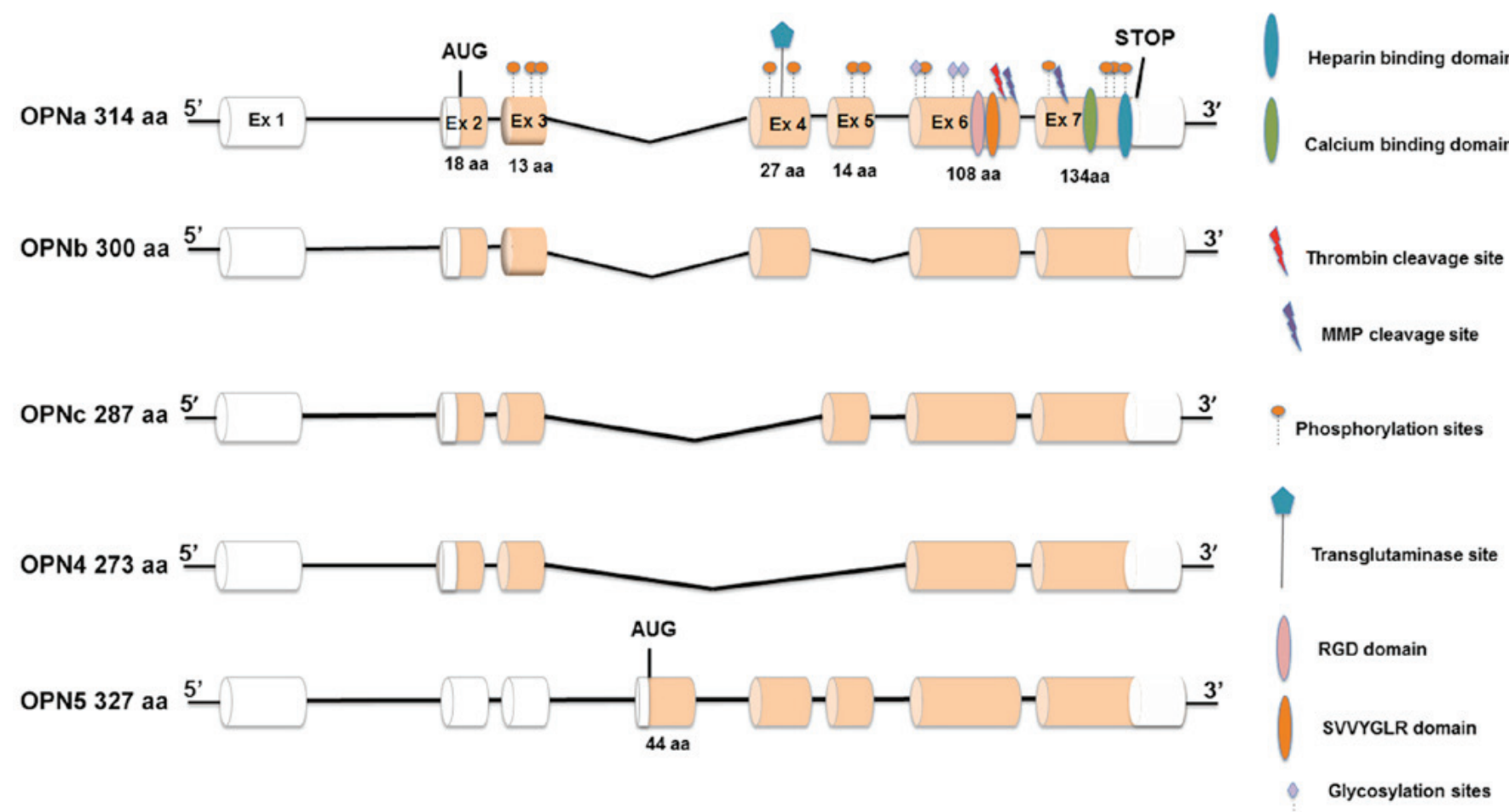

Figure 1. Structure of OPN isoforms. Exonic regions represented by white cylinders correspond to 5' and 3' untranslated regions. Each translated Ex is represented by orange cylinders. Ex total lengths are also presented. OPN functional domains are also presented above each Ex, such as RGD, SVVYGLR and calcium binding domain, as well as post-translational modifications, such as phosphorylation and glycosylation sites. Also presented are protein cleavage sites for thrombin and MMPs. The initiation translation codon (AUG) and the stop codon are also represented. OPNa, the full length isoform, contains Ex 2-7, whereas OPNb and OPNc lack Ex 5 and 4, respectively. Ex 4 and 5 are deleted in the OPN4 isoform, whereas OPN5 contains an extra Ex resulting from an inclusion of an intronic region located between Ex 3 and 4, corresponding with the longer isoform. Total length of each isoform of each isoform is also presented. OPN, osteopontin; aa, amino acids; MMP, metalloproteases; Ex, exon.

to present tissue and tumor specific roles $(17,37)$. Notably, the same OPN-SI might activate or inhibit tumor progression, depending on the tumor type (17,37). However, data regarding the expression and functional roles of OPN4 and OPN5 in tumor cells are scarce. One previous report described that these recently described OPN-SI are frequently co-expressed in esophageal carcinoma tissues (38), but functional studies evaluating these splice variants are still lacking.

In the present review, among the broadly known OPN roles on activating tumor progression, additional features involved in tumor resistance are highlighted. These typically include OPN functions on modulating cell survival, apoptosis and autophagy, as well as cell plasticity and sustaining stem-like properties of cancer cells (39), which are key factors associated with tumor resistance.

A molecular mechanism frequently associated with failure in the treatment of malignant carcinomas is the biological reprogramming of epithelialcellscalled epithelial mesenchymal plasticity.More recently, ithas been defined as a dynamic process implicated in epithelial-mesenchymal transition (EMT) and its reverse program, mesenchymal-epithelial transition (MET) or intermediate phenotypes, known as partial or intermediate EMT $(40,41)$. EMT renders cancer cells the ability to lose epithelial traits, while gaining mesenchymal features. During EMT, cells also acquire stem cell-like properties and are able to disseminate and colonize to distant organ sites, where they may exhibit elevated resistance to cancer therapies $(42,43)$. Aberrant activation of oncogenic signaling pathways, including Wnt $/ \beta$-catenin, hedgehog, Notch , PI3K-AKT, tumor necrosis factor- $\alpha$ and transforming growth factor (TGF)- $\beta$ has a critical role in EMT (44) and occurs during acquisition of EMT phenotype and resistance to therapy. Furthermore, induction of resistance is also mediated by several genes regulated by the TF NF- $\kappa$ B, including Bcl-2, Bcl-xL, X-linked inhibitor of apoptosis (XIAP), survivin and AKT, which have been reported to mediate chemoresistance and radioresistance in numerous types of tumor cells (45).

In the present report, current knowledge regarding the OPN roles on mediating chemoresistance and radioresistance were reviewed, which are the primary cancer treatment approaches that are currently used. The distinct mechanisms by which OPN can promote resistance to specific drugs and their associated signaling pathways are also explored. Based on these data, putative treatment approaches targeting OPN that have been proposed to overcome resistance or inhibit tumor progression, and the particular contribution of OPN splice variants on the resistant phenotype are then discussed.

\section{OPN and resistance to chemotherapy}

OPN is able to mediate resistance to distinct chemotherapeutic drugs and in several cancer types. Table I summarizes these data and the corresponding signaling pathways and molecular mechanisms involved.

In non-solid tumors, such as leukemia, OPN has been reported to mediate resistance to parthenolide and sorafenib. 


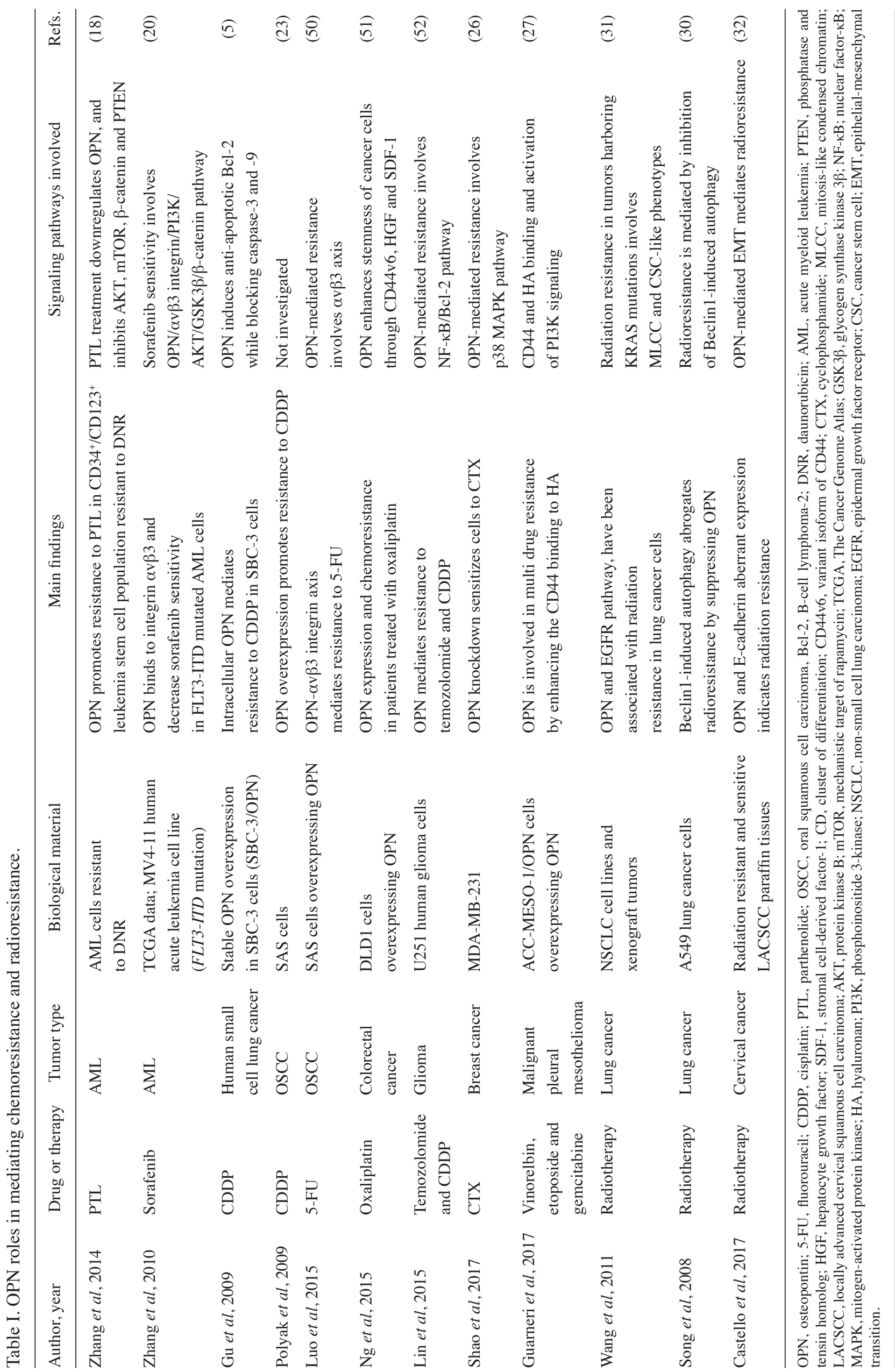


The natural compound parthenolide was demonstrated to induce apoptosis in daunorubicin-resistant stem-like leukemic cells through OPN downregulation and modulation of AKT, mTOR and $\beta$-catenin signaling (46). Particularly in acute myeloid leukemia, OPN was demonstrated to be upregulated in leukemic blasts, being correlated with a poor clinical outcome (47). Consistently, OPN promoted resistance to sorafenib by binding to $\alpha v \beta 3$ integrin and by inducing $\beta$-catenin expression in an AKT and glycogen synthase kinase (GSK) $3 \beta$-dependent manner (48).

Furthermore, there are several examples in which OPN mediates resistance in solid tumors, most of which focused on the roles of OPN in hepatocellular carcinomas (HCC). In these tumors, high OPN expression level is correlated with increased metastatic potential and resistance to taxanes or cisplatin. In lung cancer cells, intracellular OPN upregulation promotes cisplatin resistance by inducing the expression of anti-apoptotic protein Bcl-2 and by blocking caspase- 3 and caspase-9 from activation (5). Also, OPN is able to stimulate HCC cell survival and autophagy, which then can favor stem cell-like properties and the resistant phenotype in response to epirubicin and cisplatin via binding with its receptor integrin $\alpha v \beta 3$ and sustaining Forkhead box (Fox)O3a stability (42). It has been proposed that OPN may promote a cancer stem cell (CSC)-like phenotype via the $\alpha v \beta 3-\mathrm{NF}-\kappa \mathrm{B}-\mathrm{HIF}-1 \alpha$ pathway (49).

Other studies using human samples with oral squamous cell carcinoma demonstrated that OPN expression levels are correlated with therapy response and shorter overall survival. OPN upregulation in these tumors promoted resistance to cisplatin and to 5-fluorouracil and also involved the OPN-integrin $\alpha v \beta 3$ axis (50). In addition, several reports described the association between upregulated OPN expression and chemoresistance in patients treated with oxaliplatin in colorectal cancer (51), cisplatin in lung cancer (5), temozolomide and cisplatin in glioma (52), cyclophosphamide in breast cancer (53) and vinorelbin, etoposide and gemcitabine in malignant pleural mesothelioma (54). Similarly to other tumor models, resistance to these chemotherapeutic drugs mainly involves the regulated expression of octamer-binding TF 4 and sex determining region Y-box (SOX)2 (modulators of stemness), Bcl-2, caspase-3 and caspase-9 (apoptosis regulators) $(5,52)$, as well as NF- $\mathrm{NB}, \mathrm{AKT}$ and $\mathrm{p} 38 /$ mitogen-activated protein kinase (MAPK) signaling (49,51). Furthermore, it has been demonstrated in malignant pleural mesothelioma that OPN could regulate chemosensitivity to vinorelbine, etoposide and gencitabin through the alteration of CD44 binding to hyaluronate (HA) (54). HA is a linear glycosaminoglycan that interacts with cell surface receptors, including CD44, facilitating cell adhesion, cell motility, cellular proliferation and tumor progression (54). OPN is strongly involved in multidrug resistance in this tumor by enhancing the CD44 binding to HA and PI3K/AKT signaling, thereby promoting cell survival and chemoresistance (54).

\section{OPN and association to radioresistance}

In addition to OPN roles in mediating chemoresistance, reports describing the functional association between OPN and radiotherapy resistance are scarce (Table I). However, a number of reports have described the roles of OPN as a marker of response to radiotherapy $(55,56)$. OPN expression, in conjunction with the epidermal growth factor receptor pathway, have been associated with radiation resistance and poor prognosis in lung cancer, particularly in patients presenting tumors with KRAS mutations (57). It has also been reported in lung cancer that OPN is an indicator of resistance to radiotherapy. In a previous study, overexpression of beclin-1 induced cell death by autophagy in human lung cancer cells, reversing radioresistance (58). Radioresistance in these tumors has been associated with a stem cell-like phenotype and invasive potential (57). In cervical cancer, high OPN and low E-cadherin expression levels correlate with a radiation-resistant phenotype (59), further implicating OPN as a key molecule in the interface of radioresistance and cellular plasticity. Stem cell-like features and radioresistance in glioma cells can be promoted by OPN possibly via activation of CD44 signaling through its intracellular domain by enhancing HIF- $2 \alpha$ activity (60). Fig. 2 summarizes the mechanisms and signaling pathways activated by OPN on promoting chemoresistance and radioresistance.

\section{OPN in the interface of epithelial plasticity and thera- peutic resistance}

EMT corresponds with the dynamic transdifferentiation of epithelial into mesenchymal cells, in which cells lose their epithelial features and become more motile mesenchymal cells (44). Increasing evidence has demonstrated that there is a close association between EMT and resistance to therapy $(59,60)$, which may be caused by an enhancement of cancer cell survival, cell fate transition, and/or upregulation of drug resistance-related genes during the EMT transition.

The EMT process is mediated by several EMT-inducing TFs (EMT-TF), including TWIST1/2, SNAIL1/2 and zinc finger E-box binding homeobox (ZEB) $1 / 2$. In addition to these established EMT-TFs, other TFs have been demonstrated to induce or regulate EMT, including Fox TFs, GATA family, SOX, ovo-like transcriptional repressor $1 / 2$ and grainyhead-like 2 (61,62). Mechanistically, EMT-TFs suppress the expression of key epithelial markers, such as E-cadherin, while activating the expression of mesenchymal genes, such as those coding for vimentin and fibronectin. Collectively, these regulatory networks control the integrity of and the balance between the epithelial and mesenchymal phenotypes. In chemoresistant cells, EMT-TFs increase cell survival in response to therapy-induced programmed cell-death by upregulating anti-apoptotic genes, while downregulating gene products performing pro-apoptotic roles (52). Certain EMT-TFs can also contribute to hormone therapy resistance by modulating the expression of their corresponding receptors (61). These findings make the EMT process an attractive target for reducing chemotherapy resistance (61).

It has also been demonstrated that EMT-TFs act cooperatively with changes at the RNA level that regulate EMT progression, such as alternative splicing and microRNA (miRNA or miR) and long non-coding RNA mediated control of EMT (61-64). EMT process is further controlled by multiple signaling pathways, in which multiple morphogenetic and environmental signals, such as TGF- $\beta$, 


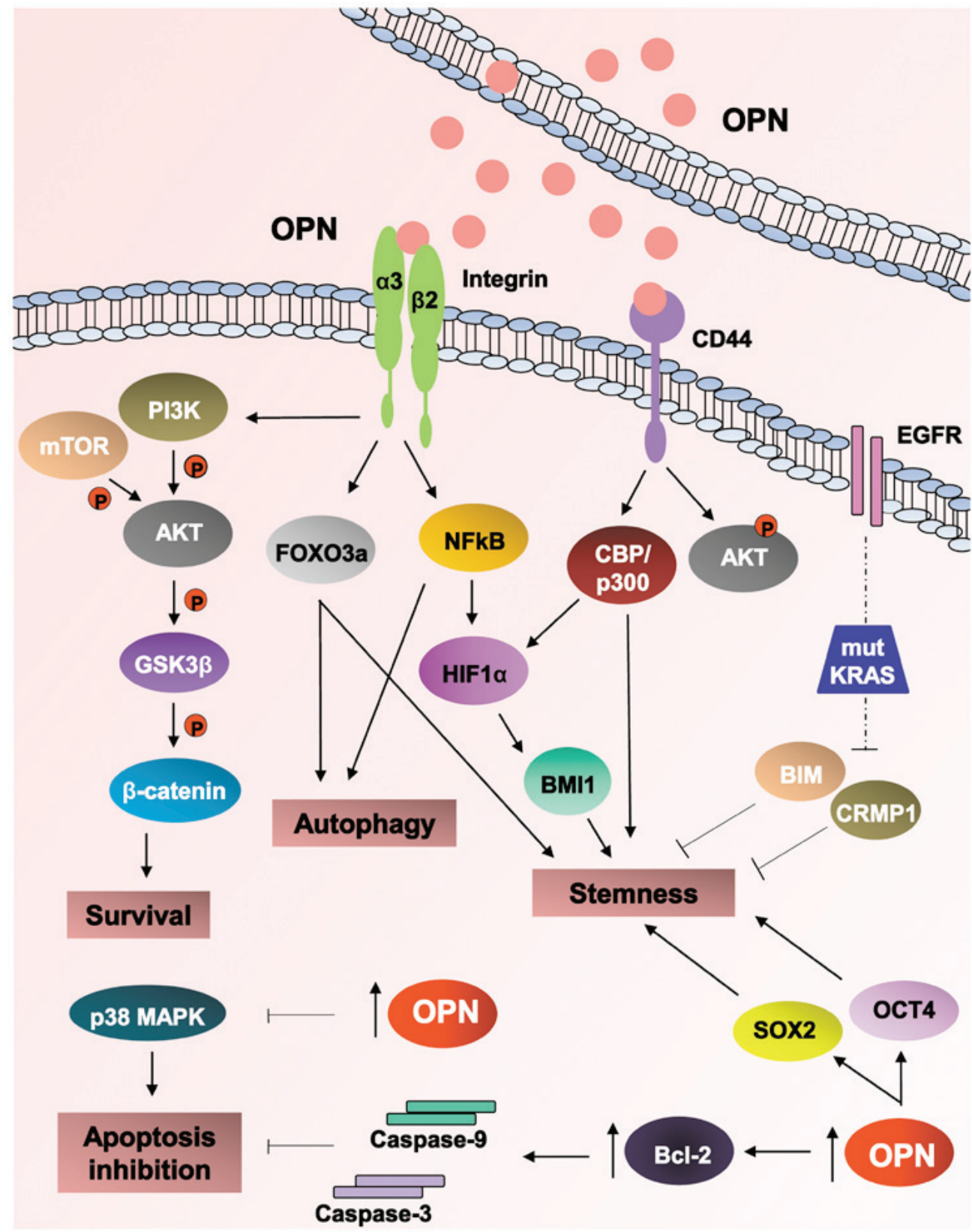

Figure 2. Cancer treatment resistance mechanisms mediated by OPN. Numerous and intricate mechanisms developed by cancer cells have been described at different levels in response to intracellular or extracellular OPN. Upon OPN binding to integrin receptors, especially by $\alpha \mathrm{V} / \beta 3 \mathrm{heterodimers,} \mathrm{the}$ $\mathrm{PI} 3 \mathrm{~K} / \mathrm{AKT} / \mathrm{mTOR} / \beta$-catenin signaling pathway is triggered, leading to cell survival and chemotherapeutic resistance. OPN can also confer stem cell-like features to cancer cells by sustaining FoxO3a stability-induced autophagy as well as activating the $\alpha / / \beta 3 / \mathrm{NF}-\mathrm{kB} / \mathrm{HIF}-1 \alpha$ pathway. Accordingly, OPN can modulate cancer stemness by altering CD44 receptor binding to hyaluronate and inducing AKT phosphorylation via CD44, thereby promoting cell survival and chemoresistance. In conjunction with the EGFR pathway, OPN expression, has been associated with radiation resistance particularly in tumors presenting KRAS mut, which was closely associated with the modulation of BIM and CRMP1 expression and thus, stem cell phenotype. Consistently, intracellular OPN was demonstrated to regulate the expression of Oct 4 and Sox 2 , well known modulators of stemness. OPN expression levels have been demonstrated to negatively regulate 38 MAPK and correlate with caspase- 3 and -9 blocking and expression of Bcl-2 anti-apoptotic protein, further contributing towards an apoptosis inactivation phenotype. Therefore, OPN has been demonstrated to modulate signaling pathways associated with chemoresistance and radioresistance, through promoting cell survival and inhibiting apoptosis as well as modulating autophagy and stemness in tumor cells. OPN, osteopontin; PI3K, phosphoinositide 3-kinase; AKT, protein kinase B; mTOR, mechanistic target of rapamycin; Fox, Forkhead box; NF-кB; nuclear factor-kB; HIF-1 $\alpha$, hypoxia-inducible factor-1 $\alpha$; Oct4, octamer-binding transcription factor 4; CD, cluster of differentiation; EGFR, epidermal growth factor receptor; mut, mutations; Bcl-2, B cell lymphoma-2; BIM, Bcl-2-like protein 11; CRMP1, collapsin response mediator protein 1; Sox2, sex determining region Y-box 2; MAPK, mitogen-activated protein kinase; GSK3 $\beta$, glycogen synthase kinase $3 \beta ;$ CBP, cAMP response element binding protein binding protein.

WNT, epidermal and platelet-derived growth factors, inflammatory cytokines and integrin receptor ligands, have been demonstrated to promote EMT in response to extracellular signals (61). Among these molecules, NF- $\mathrm{KB}$ and matrix MMPs have also been identified as specific inducers of EMT. NF- $\mathrm{kB}$ has been described as a contributor to EMT, by combining with oncogenic gene products, such as RAS in order to protect cells from apoptosis (65). MMPs induce EMT associated with malignant transformation via a pathway dependent upon production of reactive oxygen species (ROS) and degrading matrix proteins, then favoring tumor cell migration and motility (65). 
TGF- $\beta$, mostly TGF- $\beta 1$ isoform, is the most well-studied cytokine in the induction of EMT. As reviewed elsewhere (61), TGF- $\beta$ mediates EMT through mothers against decapentaplegic homolog (SMAD)-dependent or SMAD-independent pathways The SMAD-dependent pathway is initiated by binding of TGF- $\beta 1$ to TGF- $\beta$ receptor (T $\beta R) I I$ and T $\beta R I$, which then activate SMAD protein complex, which then enter the nucleus to induce the expression of lymphoid enhancer binding factor- $1 \mathrm{TF}$. This TF binds to $\beta$-catenin, suppressing transcription of epithelial markers, while promoting the expression of mesenchymal markers. SMAD complexes not only activate the expression, but also increase the activity of EMT-TFs. Other changes in gene expression during EMT occur without directly requiring EMT-TFs, but are rather controlled by TGF- $\beta$-activated SMADs, which can directly activate the expression of certain mesenchymal genes. TGF- $\beta$ also induces signaling through cytoplasmic expression of $\beta$-catenin, which is also modulated by the Wnt pathway. In addition to TGF- $\beta$-activated SMAD signals, TGF- $\beta$ also induces EMT signaling through RHO-like GTPases, PI3K/AKT and MAPK pathways. Activation of RHO, RAC and cell division control protein 42 homolog GTPases drives actin reorganization, and lamellipodia and filopodia formation. PI3K signaling activation by TGF- $\beta$ promotes the activation of mammalian TOR complex (mTORC1 and mTORC2). Notably, mTORC1 and mTORC2 modulate cell size, protein synthesis, motility and invasion. AKT decreases the level of SNAIL1 expression, attenuating E-cadherin repression and the activation of MMP-9 expression. AKT also phosphorylates GSK3 $\beta$, resulting in SNAIL-1 stabilization. TGF- $\beta$ also activates ERK, p38 and JUN N-terminal kinase/MAPK pathways, which then also increases TGF- $\beta$-induced transcription, leading to increased E-cadherin repression and activation of $\mathrm{N}$-cadherin and MMP expression. EMT signaling has been demonstrated to induce the expression of genes coding for MMP-2 and MMP-9, which cleave Type IV collagen in the basal lamina to promote post-EMT invasion of underlying tissues. MMP-3 has also been demonstrated to directly induce EMT through activation of RAC1 GTPase-reactive oxygen species signaling, which promotes SNAIL1 expression. Additionally, SNAIL1/2 can also promote breakdown of the ECM via upregulation of MMPs (61). Furthermore, MMPs are capable of degrading E-cadherin in the cell membrane. All these processes enable cells to acquire a mesenchymal phenotype (61).

In this context, OPN has previously been reported as a master regulator of epithelial-mesenchymal plasticity, once it has an important regulatory role in the expression of key EMT regulators (66-71). OPN expression also shares functional interplay with the previously mentioned traditional EMT activators, such as TGF- $\beta$, TWIST $1 / 2$, ZEB1/2 and SNAIL-family members. Importantly, OPN is able to guide EMT through specific cellular signaling pathways and by restructuring the TME to modify EMT processes (66-71).

OPN overexpression induces TWIST phosphorylation and/or activation through MAPK, AKT and/or RAC/AKT signaling pathways (71,72). Besides, OPN upregulates HIF-1 $\alpha$ to induce EMT through TWIST activation and by maintaining the tumor cell stemness (66). TWIST also serves in OPN-mediated metastasis through activation of the PI3K/AKT pathway (66). Signaling mediated by OPN interacts directly or indirectly with ZEB TF family members, as well as leading to EMT. Notably, it is known that OPN is a potent activator of NF- $\mathrm{KB}$ (73), which induces the expression of both ZEB1/2 and therefore can regulate NF- $\mathrm{BB} / \mathrm{ZEB}$ dependent EMT. Likewise, OPN can regulate ZEB-related EMT through non-NF- $\mathrm{BB}$ pathways, such as by upregulating the expression of miR-200 family members that inhibit ZEB1/2 initiated EMT (71). Otherwise, NF- $\mathrm{KB}$ has also been indicated as a key player of OPN and MMP-9 activation (74). Furthermore, OPN regulates EMT by overexpressing SNAIL EMT-TF. In addition, OPN co-regulates the expression of glioma-associated oncogene, a TF that mediates Sonic hedgehog signaling, which then induces the expression of SNAIL (71). Vimentin upregulation has also been induced by OPN (66). Notably, OPN induces the expression of TGF- $\beta$ (71).

It has been reported that OPN is able to modify the tissue and TME to support EMT and hence can also indirectly modify EMT (66). During tumor progression, limited tumor oxygen availability and tumor metabolic demands induce hypoxia. As reviewed previously $(60,61)$, the activities of HIF- $1 \alpha$ and HIF- $2 \alpha$ are enhanced in response to prolonged TGF- $\beta$ and certain tyrosine kinase receptors. HIF-1 $\alpha$ and HIF- $2 \alpha$ can then activate EMT-TF expression and/or subcellular localization in tumor cells. Immune and stromal cells from the TME are also sources of EMT-inducing cytokine stimulation. It has been demonstrated that chemotherapy and radiotherapy promote oxidative and inflammatory stress in tumor tissues, contributing to increased expression of inflammatory cytokines and subsequent induction of the EMT program. In the TME, post-translational modifications of EMT-TF can also regulate the half-life of EMT-TFs (61). Malignant signals from the TME with long-lasting effects on associated cancer cells may also modulate epithelial plasticity and then sustain the metastatic potential and tumor chemoresistance. OPN modulates tumor-specific EMT by generating cancer-associated fibroblasts (CAFs), which secrete a multitude of factors in the TME that support tumor invasiveness and metastases, including TGF- $\beta$ and interleukin-6. Tumor-derived OPN and exogenous OPN are able to induce transformation of CAFs from mesenchymal stem cells by stimulating the production of TGF- $\beta$. OPN also enhances the migration and invasion of malignant tumor cells through both the inhibition of apoptosis and by regulating the activities of MMP-2 and MMP-9, which degrade the ECM. OPN has been described to upregulate MMP-9 activity, modulating multiple signaling pathways via focal FAK, ERK and NF- $\kappa \mathrm{B}$ that regulate cytoskeletal organization, cell motility, cell growth, and also cell migration, ECM invasion and tumor growth $(61,62)$. In this context, it has been indicated that NF- $\mathrm{KB}$ may be a key player in OPN and MMP-9 activation (27). OPN is a substrate for several extracellular proteases and is cleaved in vitro and in vivo by MMPs. MMP-9, for instance, is known to cleave OPN (75), and it is also known that OPN-regulated signaling leads upregulation of MMP-2 in prostate cancer cells (74). Similarly, OPN can mediate the activation of MMP-9 during migration of prostate cancer and melanoma cells (74).

It has also been reported that OPN has an important role in regulating TGF- $\beta$-mediated processes and likely also regulates TGF- $\beta$-mediated EMT (70). Considering the emerging roles of OPN in modulating the EMT process, thus contributing to a 
drug resistant phenotype, the analysis of OPN expression has been considered as a potential target for future interventions to overcome tumor resistance. Notably, it has been reported that, unlike secreted OPN, which is able to trigger the EMT to initiate cancer metastasis, nuclear OPN is able to induce MET, contributing to metastasis establishment at secondary sites. In this model, OPN interacted with HIF-2 $\alpha$, latterly impacting on the AKT/mir-29/ZEB cascade (71). It has also demonstrated that VEGF in the TME is able to induce OPN nuclear translocation (71).

\section{OPN as a target to overcome resistance to cancer therapy}

Considering the emerging roles of OPN in several processes associated with chemoresistant and radioresistant phenotype, and its pleiotropic roles in the tumor cascade, the analysis of OPN expression and associated signaling has been considered as a potential target for future interventions trying to overcome tumor resistance, both to early and advanced tumors.

OPN-associated signaling pathways include PI3K/AKT, mTOR, $\beta$-catenin or phosphatase and tensin homolog (PTEN) gene expression (72), as well as $\alpha v \beta 3-\mathrm{NF}-\kappa \mathrm{B}-\mathrm{HIF}-1 \alpha$ (49), caspases and Bcl-2 (5,52), p38/MAPK (54) and GSK3 $\beta$ (48), among other targets. Advances in the understanding of the biology of tumor resistance, particularly the signaling pathways associated with this phenotype, may enable the development of novel approaches to overcome resistance to therapy.

Specific and targeted inhibition of these resistance-associated proteins and signaling pathways may potentially increase sensitivity of cancer cells to the cytotoxic action of chemotherapeutic agents and to radiation exposure $(44,45)$. As an example of this approach to further sensitize cells to chemotherapy by targeting OPN and associated signaling, isolated primary CD $34^{+} / \mathrm{CD} 38^{-}$bone marrow derived acute myeloid leukemia (AML) cells have been treated with curcumin and daunorubicin in combination. This strategy induced AML cell growth inhibition and increased cytotoxicity by upregulating AKT, mTOR, $\beta$-catenin or PTEN. Notably, these effects were stronger when OPN expression was specifically knocked-down (72). It has also been reported that $\mathrm{OPN} / \mathrm{NF}-\kappa \mathrm{B}$-mediated autophagy is required for the maintenance of the stemness state of pancreatic cancer cells, which is associated with survival and chemoresistance (76). These data demonstrated that the blockade of autophagy by downregulating autophagy markers or by treating these pancreatic cells with an autophagy inhibitor reduced the pancreatic CSC populations and associated features, such as the expression of CD44, CD24, CD133 and aldehyde dehydrogenase 1 (76). Once OPN is able to stimulate autophagy and the expression of CSC markers (which includes integrin and CD44 receptors), these cell populations could be prevented by OPN downregulation approaches in order to sensitize pancreatic cancer cells to current chemotherapeutic drugs, such as gemcitabine (76).

Therapeutic application of small interfering RNA molecules targeting OPN $(77,78)$ or neutralizing antibodies associated with OPN epitopes (79) have been tested in order to downregulate OPN expression levels and the results have been promising. An additional approach to sensitize cells to chemotherapy or radiotherapy would be using miRNA molecules targeting OPN or additional gene products associated with chemoresistance, such as those modulating EMT $(80,81)$, drug transporters (78) and cell survival (81). Oncogenic miRNAs are the miRNAs with a defined role in cancer. Several miRNAs are deregulated in cancer cells and correlated with tumor features. Specifically, miRNAs have been reported to influence several tumor-related processes, such as EMT, tumor invasion, metastasis and resistance to therapy $(80,82)$.

Among currently tested miRNAs targeting OPN, a number are able to modulate tumorigenicity, tumor growth and metastasis, such as miR-127-5p (83), hsa-miR-299-5p (80) and miR-181a (84). However, to the best of our knowledge, there is no report describing the specific effects of miRNAs on sensitizing tumor cells to chemotherapy. Conversely, a number of miRNAs that, via targeting OPN, may be promising tools to regulate chemoresistance and radioresistance mediated by OPN. It has been demonstrated that miR-127-5p and hsa-miR-299-5p are able to regulate OPN expression and can respectively modulate human chondrocyte cell proliferation and tumorigenicity, and also display vasculogenic mimicry of spheroid-forming breast cancer cells (80). Similarly, miR-181a regulation of OPN expression provides a novel mechanism of suppressing metastasis in cancer cell lines (84). Furthermore, three lentiviral vectors encoding miRNA against OPN have been reported to inhibit tumor growth and metastasis of human hepatocellular carcinoma, by decreasing MMP-2 and uPA expression, thus leading to inhibition of lung metastasis (85). Furthermore, RNA aptamers have also been proposed to target OPN and it has been demonstrated to decrease EMT and tumor growth $(86,87)$.

Future studies aiming to improve the effects of chemotherapy and radiotherapy could propose similar strategies targeting OPN signaling and additional pathways associated with cancer cell survival and resistance.

\section{OPN splice variants and their potential role in tumor resistance}

Although previous studies have reported the expression and roles of OPN-SI regarding distinct aspects of tumor progression, data reporting the association between their expression and resistance to therapy are limited. A recent report proposed that $\mathrm{OPNb}$ and OPNc splicing isoforms are aberrantly expressed in leukemia cells in response to distinct chemotherapeutic drugs, such as daunorubicin, idarubicin and cytarabine, further mediating resistance to these drugs (88). However, it was not clearly demonstrated that specific knockdown of these splice variants reverted chemoresistance. Our group pioneered the studies of OPN-SI and their relation to chemoresistance, demonstrating that prostate cancer cells that ectopically overexpress OPNb or OPNc are more resistant to docetaxel (DXT) and display higher survival rates (89). The DXT-resistant phenotype was also associated with EMT features in which cells overexpressing OPNb or OPNc exhibited upregulated mesenchymal markers, as opposed to epithelial markers (89). In summary, these data demonstrated that OPN-SI differently modulate chemoresistance. As nuclear OPNc has been demonstrated as a prognostic marker in breast cancer (90) and also reported to be correlated with relapse $(90,91)$ and 
poor survival (92), it is possible that nuclear OPNc may also be a potential marker of response to chemotherapy or radiotherapy, as has been previously reported (90). Future studies should further investigate the roles of OPN-SI in chemoresistance in distinct tumor models and also explore their involvement in radioresistance.

\section{Conclusions}

Growing evidence has pointed to the crucial role that OPN has in many aspects of cancer progression, including the acquisition of drug resistance. OPN not only induces integrin receptor-mediated oncogenic signaling pathways but also modulates the epithelial-mesenchymal phenotype and stemness, conferring cancer cells the ability to survive, proliferate, evade from cell death, migrate and colonize other tissues. Consequently, OPN-overexpressing cells are refractory to current treatment options as well as exhibit invasive and metastatic potential. Together, these findings provide evidence that OPN-triggered signaling pathways can be targeted to specifically induce cell death in chemo- and radio-insensitive cancer cells in order to overcome the therapeutic resistant phenotype. Future studies will uncover the role of specific OPN isoforms in the acquisition of treatment resistance and also address whether OPN and its isoforms may be reliable markers for cancer progression and poor response to standard therapy.

\section{Acknowledgments}

Not applicable.

\section{Funding}

The present study has been funded by FAPERJ (grant nos. E-26/ 210.394/2014, E-26/010.002007/2014 and E-26/203.204/2015), CNPq (grant no. 310591/2014-7); Ministério da Sáude and UFF/Proppi (L'ORÉAL-UNESCO-ABC for Women in Science).

\section{Availability of data and materials}

Not applicable.

\section{Authors' contributions}

ERPG designed the manuscript, provided financial support, wrote the manuscript, prepared figures, edited and performed major revisions. MCB contributed to figure preparation and revision, as well as writing and editing the manuscript. GNM provided financial support, prepared figures, and contributed to writing and editing the manuscript.

\section{Ethics approval and consent to participate}

Not applicable.

\section{Patient consent for publication}

Not applicable.

\section{Competing interests}

The authors declare that they have no competing interests.

\section{References}

1. Gottesman MM, Lavi O, Hall MD and Gillet J-P: Toward a better understanding of the complexity of cancer drug resistance. Annu Rev Pharmacol Toxicol 56: 85-102, 2016.

2. Bergman PJ and Harris D: Radioresistance, chemoresistance, and apoptosis resistance. The past, present, and future. Vet Clin North Am Small Anim Pract 27: 47-57, 1997.

3. Shevde LA and Samant RS: Role of osteopontin in the pathophysiology of cancer. Matrix Biol 37: 131-141, 2014.

4. Clemente N, Raineri D, Cappellano G, Boggio E, Favero F Soluri MF, Dianzani C, Comi C, Dianzani U and Chiocchetti A: Osteopontin bridging innate and adaptive immunity in autoimmune diseases. J Immunol Res 2016: 7675437, 2016.

5. Gu T, Ohashi R, Cui R, Tajima K, Yoshioka M, Iwakami S, Sasaki S, Shinohara A, Matsukawa T, Kobayashi J, et al: Osteopontin is involved in the development of acquired chemoresistance of cisplatin in small cell lung cancer. Lung Cancer 66: 176-183, 2009.

6. Huang RH, Quan YJ, Chen JH, Wang TF, Xu M, Ye M, Yuan H, Zhang CJ, Liu XJ and Min ZJ: Osteopontin promotes cell migration and invasion, and inhibits apoptosis and autophagy in colorectal cancer by activating the p38 MAPK signaling pathway. Cell Physiol Biochem 41: 1851-1864, 2017.

7. Wu Y, Jiang W, Wang Y, Wu J, Saiyin H, Qiao X, Mei X, Guo B, Fang X, Zhang L, et al: Breast cancer metastasis suppressor 1 regulates hepatocellular carcinoma cell apoptosis via suppressing osteopontin expression. PLoS One 7: e42976, 2012.

8. Wu XL, Lin KJ, Bai AP, Wang WX, Meng XK, Su XL, Hou MX, Dong PD, Zhang JJ, Wang ZY, et al: Osteopontin knockdown suppresses the growth and angiogenesis of colon cancer cells. World J Gastroenterol 20: 10440-10448, 2014.

9. Shi Z, Wang B, Chihanga T, Kennedy MA and Weber GF: Energy metabolism during anchorage-independence. Induction by osteopontin-c. PLoS One 9: e105675, 2014.

10. Malaponte G, Hafsi S, Polesel J, Castellano G, Spessotto P, Guarneri C, Canevari S, Signorelli SS, McCubrey JA and Libra M: Tumor microenvironment in diffuse large B-cell lymphoma: Matrixmetalloproteinases activation is mediated by osteopontin overexpression. Biochim Biophys Acta 1863: 483-489, 2016.

11. Caputo S and Bellone M: Osteopontin and the immune system: Another brick in the wall. Cell Mol Immunol 15: 405-407, 2018.

12. Mohammadi S, Ghaffari SH, Shaiegan M,Zarif MN, Nikbakht M, Akbari Birgani S, Alimoghadam K and Ghavamzadeh A: Acquired expression of osteopontin selectively promotes enrichment of leukemia stem cells through AKT/mTOR/PTEN/ $\beta$-catenin pathways in AML cells. Life Sci 152: 190-198, 2016.

13. Bellahcène $\mathrm{A}$, Castronovo V, Ogbureke KUE, Fisher LW and Fedarko NS: Small integrin-binding ligand N-linked glycoproteins (SIBLINGs): Multifunctional proteins in cancer. Nat Rev Cancer 8: 212-226, 2008.

14. Phillips RJ, Helbig KJ, Van der Hoek KH, Seth D and Beard MR: Osteopontin increases hepatocellular carcinoma cell growth in a CD44 dependant manner. World J Gastroenterol 18: 3389-3399, 2012.

15. Rabenstein M, Vay SU, Flitsch LJ, Fink GR, Schroeter M and Rueger MA: Osteopontin directly modulates cytokine expression of primary microglia and increases their survival. J Neuroimmunol 299: 130-138, 2016.

16. Cook AC, Tuck AB, McCarthy S, Turner JG, Irby RB, Bloom GC, Yeatman TJ and Chambers AF: Osteopontin induces multiple changes in gene expression that reflect the six 'hallmarks of cancer' in a model of breast cancer progression. Mol Carcinog 43: 225-236, 2005.

17. Gimba ER and Tilli TM: Human osteopontin splicing isoforms: Known roles, potential clinical applications and activated signaling pathways. Cancer Lett 331: 11-17, 2013.

18. Zhang H, Guo M, Chen JH, Wang Z, Du XF, Liu PX and Li WH: Osteopontin knockdown inhibits $\alpha v, \beta 3$ integrin-induced cell migration and invasion and promotes apoptosis of breast cancer cells by inducing autophagy and inactivating the PI3K/Akt/mTOR pathway. Cell Physiol Biochem 33: 991-1002, 2014. 
19. Yang L, Wei L, Zhao W, Wang X, Zheng G, Zheng M, Song X and Zuo W: Down-regulation of osteopontin expression by RNA interference affects cell proliferation and chemotherapy sensitivity of breast cancer MDA-MB-231 cells. Mol Med Rep 5: 373-376, 2012.

20. Zhang A, Liu Y, Shen Y, Xu Y and Li X: Osteopontin silencing by small interfering RNA induces apoptosis and suppresses invasion in human renal carcinoma Caki-1 cells. Med Oncol 27: 1179-1184, 2010.

21. Naor D, Wallach-Dayan SB, Zahalka MA and Sionov RV: Involvement of CD44, a molecule with a thousand faces, in cancer dissemination. Semin Cancer Biol 18: 260-267, 2008.

22. De Wever O, Demetter P, Mareel M and Bracke M: Stromal myofibroblasts are drivers of invasive cancer growth. Int J Cancer 123: 2229-2238, 2008

23. Polyak K, Haviv I and Campbell IG: Co-evolution of tumor cells and their microenvironment. Trends Genet 25: 30-38, 2009.

24. Kale S, Raja R, Thorat D, Soundararajan G, Patil TV and Kundu GC: Osteopontin signaling upregulates cyclooxygenase-2 expression in tumor-associated macrophages leading to enhanced angiogenesis and melanoma growth via $\alpha 9 \beta 1$ integrin. Oncogene 33: 2295-2306, 2014.

25. Lin CN, Wang CJ, Chao YJ, Lai M-D and Shan Y-S: The significance of the co-existence of osteopontin and tumor-associated macrophages in gastric cancer progression. BMC Cancer 15: 128, 2015.

26. Shao L, Zhang B, Wang L, Wu L, Kan Q and Fan K: MMP-9-cleaved osteopontin isoform mediates tumor immune escape by inducing expansion of myeloid-derived suppressor cells. Biochem Biophys Res Commun 493: 1478-1484, 2017.

27. Guarneri C, Bevelacqua V, Polesel J, Falzone L, Cannavò PS Spandidos DA, Malaponte G and Libra M: NF-кB inhibition is associated with OPN/MMP 9 downregulation in cutaneous melanoma. Oncol Rep 37: 737-746, 2017.

28. Liu GX, Sun JT, Yang MX, Qi XM, Shao QQ, Xie Q, Qu X Wei FC and Sun SZ: OPN promotes survival of activated T cells by up-regulating CD44 in patients with oral lichen planus. Clin Immunol 138: 291-298, 2011.

29. Matušan-Ilijaš K, Damante G, Fabbro D, Dorđević G, Hadžisejdić I, Grahovac M, Marić I, Spanjol J, Grahovac B Jonjić $\mathrm{N}$, et al: Osteopontin expression correlates with nuclear factor- $\kappa \mathrm{B}$ activation and apoptosis downregulation in clear cell renal cell carcinoma. Pathol Res Pract 207: 104-110, 2011.

30. Song G, Cai QF, Mao YB, Ming YL, Bao SD and Ouyang GL: Osteopontin promotes ovarian cancer progression and cell survival and increases HIF-1alpha expression through the PI3-K/ Akt pathway. Cancer Sci 99: 1901-1907, 2008.

31. Wang HL, Ruan LH and Zhao XQ: Expression of osteopontin and VEGF in acute leukemia and their relationship with angiogenesis. Zhongguo Shi Yan Xue Ye Xue Za Zhi 19: 926-929, 2011 (In Chinese)

32. Castello LM, Raineri D, Salmi L, Clemente N, Vaschetto R, Quaglia M, Garzaro M, Gentilli S, Navalesi P, Cantaluppi V, et al: Osteopontin at the crossroads of inflammation and tumor progression. Mediators Inflamm 2017: 4049098, 2017.

33. Dai J, Peng L, Fan K, Wang H, Wei R, Ji G, Cai J, Lu B, Li B, Zhang $\mathrm{D}$, et al: Osteopontin induces angiogenesis through activation of PI3K/AKT and ERK1/2 in endothelial cells. Oncogene 28: 3412-3422, 2009.

34. Chakraborty G, Jain S and Kundu GC: Osteopontin promotes vascular endothelial growth factor-dependent breast tumo growth and angiogenesis via autocrine and paracrine mechanisms. Cancer Res 68: 152-161, 2008.

35. Tilli TM, Mello KD, Ferreira LB, Matos AR, Accioly MT, Faria PA, Bellahcène A, Castronovo V and Gimba ER: Both osteopontin-c and osteopontin-b splicing isoforms exert pro-tumorigenic roles in prostate cancer cells. Prostate 72: 1688-1699, 2012.

36. Nuñez-GarciaM,Gomez-SantosB,BuquéX,García-RodriguezJL, Romero MR, Marin JJG, Arteta B, García-Monzón C, Castaño L, Syn WK, et al: Osteopontin regulates the cross-talk between phosphatidylcholine and cholesterol metabolism in mouse liver. J Lipid Res 58: 1903-1915, 2017.

37. Briones-Orta MA, Avendaño-Vázquez SE, Aparicio-Bautista DI, Coombes JD, Weber GF and Syn W-K: Osteopontin splice variants and polymorphisms in cancer progression and prognosis. Biochim Biophys Acta Rev Cancer 1868: 93-108, 108.A, 2017.

38. Lin J, Myers AL, Wang Z, Nancarrow DJ, Ferrer-Torres D, Handlogten A, Leverenz K, Bao J, Thomas DG, Wang TD, et al: Osteopontin (OPN/SPP1) isoforms collectively enhance tumor cell invasion and dissemination in esophageal adenocarcinoma. Oncotarget 6: 22239-22257, 2015.
39. Choi SI, Kim SY, Lee JH, Kim JY, Cho EW and Kim IG: Osteopontin production by TM4SF4 signaling drives a positive feedback autocrine loop with the STAT3 pathway to maintain cancer stem cell-like properties in lung cancer cells. Oncotarget 8: 101284-101297, 2017.

40. Sui H, Zhu L, Deng W and Li Q: Epithelial-mesenchymal transition and drug resistance: Role, molecular mechanisms, and therapeutic strategies. Oncol Res Treat 37: 584-589, 2014.

41. Ye X and Weinberg RA: Epithelial-mesenchymal plasticity: A central regulator of cancer progression. Trends Cell Biol 25: 675-686, 2015.

42. Liu X and Fan D: The epithelial-mesenchymal transition and cancer stem cells: Functional and mechanistic links. Curr Pharm Des 21: 1279-1291, 2015.

43. Li L and Li W: Epithelial-mesenchymal transition in human cancer: Comprehensive reprogramming of metabolism, epigenetics, and differentiation. Pharmacol Ther 150: 33-46, 2015.

44. Gonzalez DM and Medici D: Signaling mechanisms of the epithelial-mesenchymal transition. Sci Signal 7: re8, 2014.

45. Li F and Sethi G: Targeting transcription factor NF-kappaB to overcome chemoresistance and radioresistance in cancer therapy. Biochim Biophys Acta 1805: 167-180, 2010.

46. Mohammadi S, Zahedpanah M, Ghaffari SH, Shaiegan M, Nikbakht $\mathrm{M}$ and Nikugoftar M: Osteopontin plays a unique role in resistance of $\mathrm{CD}_{3} 4^{+} / \mathrm{CD} 123^{+}$human leukemia cell lines KG1a to parthenolide. Life Sci 189: 89-95, 2017.

47. Liersch R, Shin JW, Bayer M, Schwöppe C, Schliemann C, Berdel WE, Mesters R and Detmar M: Analysis of a novel highly metastatic melanoma cell line identifies osteopontin as a new lymphangiogenic factor. Int J Oncol 41: 1455-1463, 2012.

48. Yi H, Zeng D, Shen Z, Liao J, Wang X, Liu Y, Zhang X and Kong $P$ : Integrin alphavbeta 3 enhances $\beta$-catenin signaling in acute myeloid leukemia harboring Fms-like tyrosine kinase-3 internal tandem duplication mutations: Implications for microenvironment influence on sorafenib sensitivity. Oncotarget 7 : 40387-40397, 2016.

49. Cao L, Fan X, Jing W, Liang Y, Chen R, Liu Y, Zhu M, Jia R, Wang $\mathrm{H}$, Zhang $\mathrm{X}$, et al: Osteopontin promotes a cancer stem cell-like phenotype in hepatocellular carcinoma cells via an integrin-NF- $\kappa$ B-HIF-1 $\alpha$ pathway. Oncotarget 6: 6627-6640, 2015.

50. Luo SD, Chen YJ, Liu CT, Rau KM, Chen YC, Tsai HT, Chen CH and Chiu TJ: Osteopontin involves cisplatin resistance and poor prognosis in oral squamous cell carcinoma. BioMed Res Int 2015: 508587, 2015.

51. Ng L, Wan T, Chow A, Iyer D, Man J, Chen G, Yau TC, Lo O, Foo CC, Poon JT, et al: Osteopontin overexpression induced tumor progression and chemoresistance to oxaliplatin through induction of stem-like properties in human colorectal cancer. Stem Cells Int 2015: 247892, 2015.

52. Qian C, Li P, Yan W, Shi L, Zhang J, Wang Y, Liu H and You Y: Downregulation of osteopontin enhances the sensitivity of glioma U251 cells to temozolomide and cisplatin by targeting the NF-кB/Bcl 2 pathway. Mol Med Rep 11: 1951-1955, 2015.

53. Pang H, Cai L, Yang Y, Chen X, Sui G and Zhao C: Knockdown of osteopontin chemosensitizes MDA-MB-231 cells to cyclophosphamide by enhancing apoptosis through activating p38 MAPK pathway. Cancer Biother Radiopharm 26: 165-173, 2011.

54. Tajima K, Ohashi R, Sekido Y, Hida T, Nara T, Hashimoto M, Iwakami S, Minakata K, Yae T, Takahashi F, et al: Osteopontinmediated enhanced hyaluronan binding induces multidrug resistance in mesothelioma cells. Oncogene 29: 1941-1951,2010.

55. Wohlleben G, Scherzad A, Güttler A, Vordermark D, Kuger S, Flentje $\mathrm{M}$ and Polat B: Influence of hypoxia and irradiation on osteopontin expression in head and neck cancer and glioblastoma cell lines. Radiat Oncol 10: 167, 2015.

56. Ostheimer C, Schweyer F, Reese T, Bache M and Vordermark D: The relationship between tumor volume changes and serial plasma osteopontin detection during radical radiotherapy of nonsmall-cell lung cancer. Oncol Lett 12: 3449-3456, 2016.

57. Wang M, Han J, Marcar L, Black J, Liu Q, Li X, Nagulapalli K, Sequist LV, Mak RH, Benes $\mathrm{CH}$, et al: Radiation resistance in KRAS-mutated lung cancer is enabled by stem-like properties mediated by an osteopontin-EGFR pathway. Cancer Res 77: 2018-2028, 2017.

58. Chang SH, Minai-Tehrani A, Shin JY, Park S, Kim JE, Yu KN, Hong SH, Hong CM, Lee KH, Beck GR Jr, et al: Beclin1-induced autophagy abrogates radioresistance of lung cancer cells by suppressing osteopontin. J Radiat Res (Tokyo) 53: 422-432, 2012. 
59. Huang X, Qian Y, Wu H, Xie X, Zhou Q, Wang Y, Kuang W, Shen L, Li K, Su J, et al: Aberrant expression of osteopontin and E-cadherin indicates radiation resistance and poor prognosis for patients with cervical carcinoma. J Histochem Cytochem 63: 88-98, 2015.

60. Pietras A, Katz AM, Ekström EJ, Wee B, Halliday JJ, Pitter KL, Werbeck JL, Amankulor NM, Huse JT and Holland EC: Osteopontin-CD44 signaling in the glioma perivascular niche enhances cancer stem cell phenotypes and promotes aggressive tumor growth. Cell Stem Cell 14: 357-369, 2014.

61. van Staalduinen J,Baker D, Ten DijkeP and van Dam H: Epithelialmesenchymal-transition-inducing transcription factors: New targets for tackling chemoresistance in cancer? Oncogene: Jul 12, 2018 (Epub ahead of print). doi: 10.1038/s41388-018-0378-x.

62. Shibue T and Weinberg RA: EMT, CSCs, and drug resistance: The mechanistic link and clinical implications. Nat Rev Clin Oncol 14: 611-629, 2017.

63. Vega S, Morales AV, Ocaña OH, Valdés F, Fabregat I and Nieto MA: Snail blocks the cell cycle and confers resistance to cell death. Genes Dev 18: 1131-1143, 2004.

64. Lamouille S, Xu J and Derynck R: Molecular mechanisms of epithelial-mesenchymal transition. Nat Rev Mol Cell Biol 15: 178-196, 2014.

65. Cichon MA and Radisky DC: ROS-induced epithelial-mesenchymal transition in mammary epithelial cells is mediated by $\mathrm{NF}-\kappa \mathrm{B}-$ dependent activation of Snail. Oncotarget 5: 2827-2838, 2014.

66. Kothari AN, Arffa ML, Chang V, Blackwell RH, Syn WK, Zhang J, Mi Z and Kuo PC: Osteopontin-A Master Regulator of Epithelial-Mesenchymal Transition. J Clin Med 5: 5, 2016.

67. Yu X, Zheng Y, Zhu X, Gao X, Wang C, Sheng Y, Cheng W, Qin L, Ren N, Jia H, et al: Osteopontin promotes hepatocellular carcinoma progression via the PI3K/AKT/Twist signaling pathway. Oncol Lett 16: 5299-5308, 2018.

68. Li NY, Weber CE, Mi Z, Wai PY, Cuevas BD and Kuo PC: Osteopontin up-regulates critical epithelial-mesenchymal transition transcription factors to induce an aggressive breast cancer phenotype. J Am Coll Surg 217: 17-26, 2013.

69. Dong Q, Zhu X, Dai C, Zhang X, Gao X, Wei J, Sheng Y, Zheng Y, Yu J, Xie L, et al: Osteopontin promotes epithelialmesenchymal transition of hepatocellular carcinoma through regulating vimentin. Oncotarget 7: 12997-13012, 2016.

70. Weber CE, Li NY, Wai PY and Kuo PC: Epithelial-mesenchymal transition, TGF- $\beta$, and osteopontin in wound healing and tissue remodeling after injury. J Burn Care Res 33: 311-318, 2012.

71. Jia R, Liang Y, Chen R, Liu G, Wang H, Tang M, Zhou X, Wang $\mathrm{H}$, Yang $\mathrm{Y}$, Wei $\mathrm{H}$, et al: Osteopontin facilitates tumor metastasis by regulating epithelial-mesenchymal plasticity. Cell Death Dis 7: e2564, 2016

72. Zahed Panah M, Nikbakht M, Sajjadi SM, Rostami S, Norooznezhad AH, Kamranzadeh Fumani H, Ghavamzadeh A and Mohammadi S: Anti-apoptotic effects of osteopontin via the up-regulation of $\mathrm{AKT} / \mathrm{mTOR} / \beta$-catenin loop in acute myeloid leukemia cells. Int J Hematol Oncol Stem Cell Res 11: 148-157, 2017

73. Li X, Jiang Z, Li X and Zhang X: SIRT1 overexpression protects non-small cell lung cancer cells against osteopontin-induced epithelial-mesenchymal transition by suppressing $N F-\kappa B$ signaling. OncoTargets Ther 11: 1157-1171, 2018.

74. Castellano G, Malaponte G, Mazzarino MC, Figini M, Marchese F, Gangemi P, Travali S, Stivala F, Canevari S and Libra M: Activation of the osteopontin/matrix metalloproteinase-9 pathway correlates with prostate cancer progression Clin Cancer Res 14: 7470-7480, 2008

75. Tan TK, Zheng G, Hsu TT, Lee SR, Zhang J, Zhao Y, Tian X, Wang Y, Wang YM, Cao Q, et al: Matrix metalloproteinase-9 of tubular and macrophage origin contributes to the pathogenesis of renal fibrosis via macrophage recruitment through osteopontin cleavage. Lab Invest 93: 434-449, 2013.
76. Yang MC, Wang HC, Hou YC, Tung HL, Chiu TJ and Shan YS: Blockade of autophagy reduces pancreatic cancer stem cell activity and potentiates the tumoricidal effect of gemcitabine. Mol Cancer 14: 179, 2015.

77. Huang G, Du M-Y, Zhu H, Zhang N, Lu ZW, Qian LX, Zhang W, Tian X, He X and Yin L: MiRNA-34a reversed TGF- $\beta$-induced epithelial-mesenchymal transition via suppression of SMAD4 in NPC cells. Biomed Pharmacother 106: 217-224, 2018.

78. Cui H, Zhang AJ, Chen M and Liu JJ: ABC transporter inhibitors in reversing multidrug resistance to chemotherapy. Curr Drug Targets 16: 1356-1371, 2015.

79. Zhang F, Luo W, Li Y, Gao S and Lei G: Role of osteopontin in rheumatoid arthritis. Rheumatol Int 35: 589-595, 2015.

80. Shevde LA, Metge BJ, Mitra A, Xi Y, Ju J, King JA and Samant RS: Spheroid-forming subpopulation of breast cancer cells demonstrates vasculogenic mimicry via hsa-miR-299-5p regulated de novo expression of osteopontin. J Cell Mol Med 14: 1693-1706, 2010

81. Bhattacharya SD, Mi Z, Kim VM, Guo H, Talbot LJ and Kuo PC: Osteopontin regulates epithelial mesenchymal transitionassociated growth of hepatocellular cancer in a mouse xenograft model. Ann Surg 255: 319-325, 2012.

82. Wang J, Yang M, Li Y and Han B: The role of microRNAs in the chemoresistance of breast cancer. Drug Dev Res 76: 368-374, 2015.

83. Liang J, Xu L, Zhou F, Liu AM, Ge HX, Chen YY and Tu M: MALAT1/miR-127-5p regulates osteopontin (OPN)-mediated proliferation of human chondrocytes through PI3K/Akt pathway. J Cell Biochem 119: 431-439, 2018.

84. Boguslawska J, Sokol E, Rybicka B, Czubaty A, Rodzik K and Piekielko-Witkowska A: microRNAs target SRSF7 splicing factor to modulate the expression of osteopontin splice variants in renal cancer cells. Gene 595: 142-149, 2016.

85. Sun BS, Dong QZ, Ye QH, Sun HJ, Jia HL, Zhu XQ, Liu DY, Chen J, Xue Q, Zhou HJ, et al: Lentiviral-mediated miRNA against osteopontin suppresses tumor growth and metastasis of human hepatocellular carcinoma. Hepatology 48: 1834-1842, 2008.

86. Hunter C, Bond J, Kuo PC, Selim MA and Levinson H: The role of osteopontin and osteopontin aptamer (OPN-R3) in fibroblast activity. J Surg Res 176: 348-358, 2012.

87. Talbot LJ, Mi Z, Bhattacharya SD, Kim V, Guo H and Kuo PC: Pharmacokinetic characterization of an RNA aptamer against osteopontin and demonstration of in vivo efficacy in reversing grow th of human breast cancer cells. Surgery 150: 224-230, 2011

88. Mirzaei A, Mohammadi S, Ghaffari SH, Nikbakht M, Bashash D, Alimoghaddam K and Ghavamzadeh A: Osteopontin b and c isoforms: Molecular Candidates Associated with Leukemic Stem Cell Chemoresistance in Acute Myeloid Leukemia. Asian Pac J Cancer Prev 18: 1707-1715, 2017

89. Nakamura KDM, Tilli TM, Wanderley JL, Palumbo A Jr, Mattos RM, Ferreira AC, Klumb CE, Nasciutti LE and Gimba ER: Osteopontin splice variants expression is involved on docetaxel resistance in PC3 prostate cancer cells. Tumour Biol 37: 2655-2663, 2016

90. Zduniak K, Ziolkowski P, Ahlin C, Agrawal A, Agrawal S, Blomqvist C, Fjällskog ML and Weber GF: Nuclear osteopontin-c is a prognostic breast cancer marker. Br J Cancer 112: 729-738, 2015.

91. Ortiz-Martínez F, Perez-Balaguer A, Ciprián D, Andrés L, Ponce J, Adrover E, Sánchez-Payá J, Aranda FI, Lerma E and Peiró G: Association of increased osteopontin and splice variant-c mRNA expression with HER2 and triple-negative/basal-like breast carcinomas subtypes and recurrence. Hum Pathol 45: 504-512, 2014

92. Patani N, Jiang W and Mokbel K: Osteopontin C mRNA expression is associated with a poor clinical outcome in human breast cancer. Int J Cancer 122: 2646, 2008. 